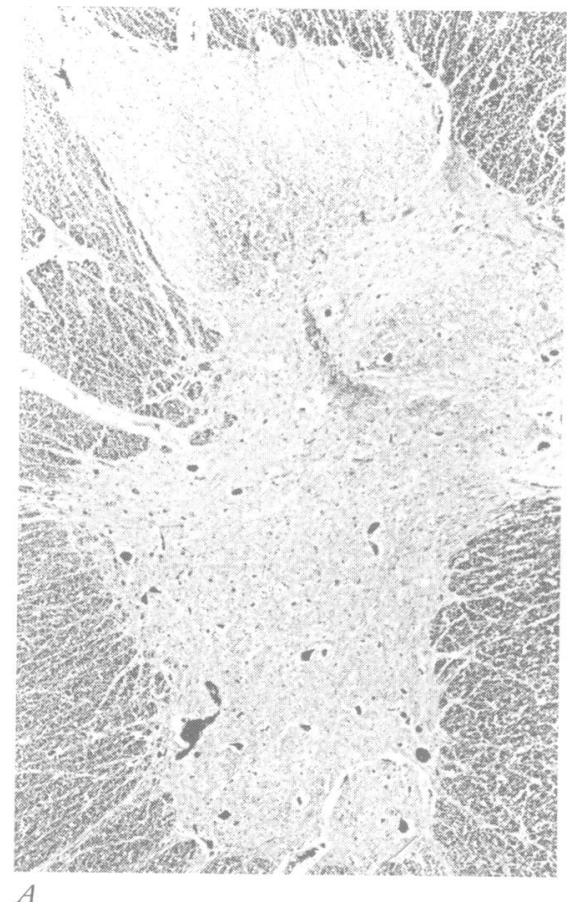

A

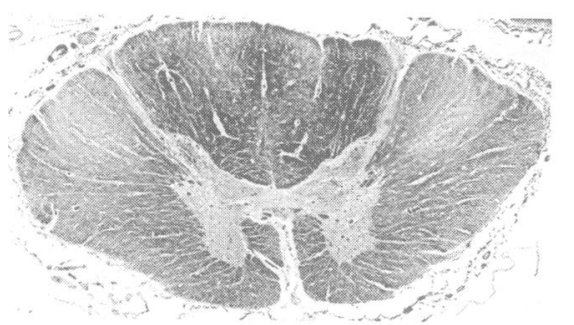

$B$

Figure 2 (A) Neuronal loss is apparent in the intermediolateral column, Clarke's column, and anterior horn of the thoracic spine (cresyl violet/Luxol fast blue $\times$ 80). (B) Myelin pallor in the pyramidal tracts of the thoracic spine is accompanied by symmetric patches of myelin loss in the posterior columns (cresyl violet/Luxol fast blue $\times 22$ ).

There was myelin pallor of the lateral corticospinal tracts and posterior columns, and the latter assumed the symmetric butterfly shape of the middle zone (fig 2B). Spinocerebellar tract degeneration was noted at the level of the pyramidal decussation. The posterior root ganglia were normal. The cervical sympathetic ganglia showed no apparent neuronal loss. Increases in satellite cells were not noticed. A few Lewy bodies and round or elongated eosinophilic bodies were occasionally encountered in the interstitium. Immunohistochemically, hyaline inclusions in $\alpha$-motor neurons of the brainstem and spinal cord, and eosinophilic bodies in the cervical sympathetic ganglia were stained positive for ubiquitin. A few intraneuronal conglomerates appeared to stain in a granular fashion. Spheroids and conglomerates were positive for neurofilaments.

This patient had pure autonomic failure with the features of motor neuron disease, which is not usually complicated by primary autonomic failure. The patient clinically showed autonomic failure, mainly manifested by orthostatic hypotension. The low resting noradrenaline concentration and impaired heart rate response to atropine with low mean R-R interval of ECG suggested dysfunction of the postganglionic adrenergic and the cardiac parasympathetic system respectively. Thus the whole clinical feature of the patient could be diagnosed as pure autonomic failure, which chiefly concerned the cardiovascular system. The main pathological changes were found in the dorsal vagal nuclei and intermediolatera columns, which were considered to be the neuroanatomical system with the predominant involvement of the degenerative process. Along with the existence of eosinophilic bodies in the sympathetic ganglia, these findings were similar to those described in pure autonomic failure. ${ }^{23} \mathrm{By}$ contrast with the previously reported cases, this patient showed the absence of neuronal loss or Lewy bodies in the substantia nigra and locus coeruleus. This suggests that pure autonomic failure could be recognised as a clinicopathological entity separated from multiple system atrophy or Parkinson's disease.

Although motor neuron disease does not usually coexist with autonomic failure, our patient exhibited loss of anterior horn cells and pyramidal tract degeneration. In addition, we found symmetric patches of myelin loss in the posterior columns, degeneration in the spinocerebellar tracts, and loss of cells from Clarke's columns. This type of distribution is similar to that reported in cases of a familial form of motor neuron disease. ${ }^{4}$ Furthermore, Lewy body-like hyaline inclusions and swollen cord-like axons, which were both noted in our patient, have often been described in these familial cases. Whether a familial form of motor neuron disease constitutes a distinct disease entity or not, our patient shares its characteristics.

The autonomic nervous system may be affected in motor neuron disease, with-for example, a mild degree of neuronal loss in the intermediolateral columns. ${ }^{5}$ However, out patient showed the obvious neurodegenerative changes reported in pure autonomic failure as well as those described in a familial form of motor neuron disease. Therefore this case shows an unusual constellation of pure autonomic failure and motor neuron disease.

We thank Dr M Obata and Dr S Fukuyama for helping evaluate the autonomic nervous system.

K NODA

Department of Internal Medicine, Fukushima Seikyo Hospital, Hiroshima, fapan S KATAYAMA C WATANABE Y YAMAMURA

S Nird Department of Intermal Medicine
S YONEHARA K INAI

Second Department of Pathology, School of Medicine, Hiroshima University, Hiroshima, fapan Correspondence to: $\mathrm{Dr} \mathrm{K}$ Noda, Division of
Internal Medicine, Fukushima Seikyo Hospital, 42-7, Miyakomachi, Nishiku, Hiroshima-shi, 733, 42-7,
Japan.

1 Bannister R, Mathias CJ. Introduction and classification of autonomic disorders. In:
Bannister R, Mathias CJ, eds. Autonomic failure. A textbook of clinical disorders of the auto nomic nervous system. 3rd ed. Oxford: Oxford University Press, 1992:1-12.

2 Johnson RH, Lee G de J, Oppenheimer DR, Spalding JMK. Autonomic failure with orthostatic hypotension due to intermediolateral column degeneration cases with autopsies. $Q \mathcal{F}$ Med $1966 ; 35$ : 276-92.

3 Roessmann U, van den Noort S, McFarland DE. Idiopathic orthostatic hypotension. Arch Neurol 1971;24:503-10.

4 Hirano A, Kurland LT, Sayre GP. Familial amyotrophic lateral sclerosis: a subgroup characterized by posterior and spinocerebel- lar tract involvement and hyaline inclusions in the anterior horn cells. Arch Neurol 1967; 16:232-43.

5 Kennedy PGE, Duchen LW. A quantitative study of intermediolateral column cells in syndrome. I Neurol Neurosurg Psychiatry 1985;48:1103-6.

\section{Glutaric aciduria type 1 in adulthood}

Glutaric aciduria type 1 (GA-1) is an autosomal recessive disorder caused by deficiency of the mitochondrial enzyme glutaryl CoA dehydrogenase, which oxidises and carboxylates glutaryl CoA, an intermediate step in the metabolism of lysine and tryptophan. ${ }^{1}$ The onset of clinical manifestations is usually within the first year of life with an acute encephalopathic illness, often triggered by intercurrent infection. A severe dystonic-dyskinetic syndrome develops in most patients, but others are less severely affected and asymptomatic cases have been described. It is a rare condition with only about 100 cases reported in the medical literature since its original description in $1975 .^{2}$ However, it has been suggested that GA-1 is underdiagnosed and may exist undetected in populations of children and adults labelled as having cerebral palsy. ${ }^{23}$ We report a patient with GA-1 in whom the diagnosis was made at the age of 50 years when she was referred for reassessment of chronic neurological disability.

The patient was the product of a full term normal delivery and there were no problems in the neonatal period. Motor development was mildly delayed, and she did not start to walk until the age of 18 months when she was noted to drag her right leg. At the age of 7 years she was admitted to hospital with a "paralytic illness". She remained in hospital for four months and at the time of discharge required callipers to walk. Her manual dexterity was poor and her speech was slurred. These neurological disabilities subsequently remained stable. At the age of 12 a right subtalar fusion was performed, and eight years later the left femur was shortened. She was able to complete her education at a normal school and then worked for 10 years in a factory before getting married. She was referred to us at the age of 50 because of increasing difficulty in walking caused by pain in the right ankle. There were no new neurological symptoms and no symptoms of autonomic dysfunction. She was being treated with a non-steroidal anti-inflammatory drug and was on hormone replacement therapy. She had not received any neuroleptic medication. Both her parents were caucasian and had died in their 80s. They had had no neurological illnesses, and there was no consanguinity. The patient has two siblings and three children of her own, all of whom are well with no neurological disorder.

Examination showed a severe dysarthria which made her speech very difficult to comprehend. Psychometric testing, however, showed her performance to be in the average range, with a verbal IQ of 93 . The non-verbal part of the WAIS-R was not performed because of limited manual dexterity, but good average scores were achieved on other tests of non-verbal reasoning. There was a left exotropia, but eye movements were otherwise normal. There was pronounced lingual dystonia and orofacial dyskinesia. Her right leg was hypoplastic and she used callipers and two sticks to walk. There 
was dystonic posturing and clawing of both hands. Power was reduced (grade 4) at the wrist and in the hands, worse on the right. The right leg was flaccid, and there was no movement at the right ankle. Power was reduced (grade 4) proximally in both legs. The right ankle jerk was absent, but all the other tendon reflexes were brisk. The righ plantar response was absent, the left extensor. There were no cerebellar or sensory signs. General examination was normal.

The following investigations were normal or negative: routine haematology and biochemistry, serum copper and caeruloplasmin, serum and urinary amino acids, syphilis serology, antinuclear factor, lupus anticoagulant, and anticardiolipin antibody. Constituents of CSF were normal including lactate $(1.15 \mathrm{mmol} / \mathrm{l}$; normal range: $0.5-1.8$ $\mathrm{mmol} / \mathrm{l})$, and pyruvate $(83 \mu \mathrm{mol} / 1$; normal range: $40-138 \mu \mathrm{mol} / \mathrm{l}$ ). Motor and sensory nerve conduction velocities and amplitudes were within normal limits.

Brain MRI showed large CSF spaces around the anterior portions of both temporal lobes (figure). There was also a mild degree of generalised cortical atrophy and focal areas of signal abnormality in the white matter of both cerebral hemispheres. A trace of glutaric acid was present in the urine, with a small peak of 3-hydroxyglutaric acid. Glutaryl CoA dehydrogenase activity, measured by release of labelled $\mathrm{CO}_{2}$ from [1,5$\left.{ }^{14} \mathrm{C}\right]$ glutaryl $\mathrm{CoA}$ by cell homogenate, was undetectable in cultured skin fibroblasts $(0 \cdot 0$ and 0.0 compared with $12.9,13.9,19.6$, $20.1,12.5$ and $12.7 \mathrm{pmol} / \mathrm{mg}$ protein $/ \mathrm{min}$ in simultaneous controls) and was $<1 \%$ of normal in blood lymphocytes $(0.06$ and 0.02 compared with 11.3 and $10.8 \mathrm{pmol} / \mathrm{mg}$ protein/min in a simultaneous control) confirming a diagnosis of GA-1.

The dystonia and dyskinesia with severe dysarthria but relative preservation of cognitive function in this patient is typical of GA$1 .^{2}$ In addition, the bitemporal enlargement of CSF spaces on MRI, although occasionally reported in other conditions, is strongly suggestive of GA-1. No other cause was identified for this patient's neurological disorder.

Most patients with GA-1 present in the first year of life with acute metabolic decompensation and encephalopathy. A recent

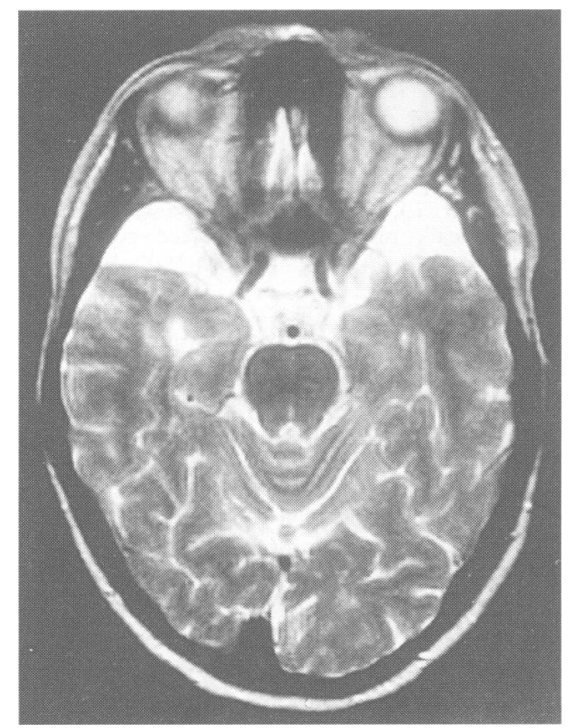

1.5 T MRI. T2 weighted axial image showing enlarged anterior temporal CSF spaces. review of reported cases suggests that $97 \%$ of those surviving an acute encephalopathic onset are left with severe disabilities. ${ }^{2}$ Our patient presented with delayed walking and dragging the right leg. Up to $25 \%$ of cases may present insidiously and there is some evidence that the prognosis in this group is better with only $64 \%$ being left with severe disability. ${ }^{2}$ It is rare for new neurological deficits to develop after the age of 3 years, and after this age any residual disability remains static. ${ }^{4}$ Our patient is unusual in that, after a gradual onset, there was further deterioration in neurological function at the age of 7 . The details of that episode are limited, but as the neurological sequelae are typical of GA-1, it seems reasonable to attribute it to GA-1 rather than to implicate a second disease process. From the age of 7 until 50, her neurological state remained stable. The current decline in mobility was thought to be related to degenerative joint disease rather than neurological deterioration.

The proportion of cases diagnosed in infancy that will survive into adulthood is, as yet, unknown given that GA-1 was first described only 20 years ago. At the age of 50 years, our patient is the oldest documented case with GA-1. Four other clinically affected patients with GA-1, in whom the diagnosis was made after the age of 18 (aged $19,23,28$, and 37), have been reported. ${ }^{34}$ It is likely that there are other unrecognised cases of GA-1, born before the condition was first described, who have survived into adulthood.

The diagnosis of GA-1 in clinically suspected cases is usually made by demonstrating excretion of glutaric acid and 3-hydroxyglutaric acid in urine. Glutaric acid is found in the urine in other metabolic disorders such as multiple acyl-CoA dehydrogenase deficiency, but the presence of 3hydroxyglutaric acid makes the diagnosis of GA-1 almost certain, as it has not been found in any other condition. ${ }^{1}$ However, during periods of neurological stability, which will invariably be the case in adults, excretion may be low or undetectable.? Neuroradiological findings (enlarged frontotemporal CSF spaces and signal abnormalities in cerebral white matter and, in severe cases, in the basal ganglia) may also suggest a diagnosis of GA-1, but they are not specific. Similar abnormalities are seen in the basal ganglia in the mitochondrial encephalopathies and in Leigh's syndrome. Although dystonia may be prominent in Leigh's syndrome, ${ }^{5}$ the presence of other features such as retinopathy, peripheral neuropathy, and dysmorphism help to distinguish it from GA-1. In addition, enlarged frontotemporal CSF spaces are not found in Leigh's syndrome. To confirm a diagnosis of GA-1, glutaryl CoA dehydrogenase activity must be measured in cultured skin fibroblasts or blood lymphocytes. ${ }^{3}$ It is usually undetectable in homozygotes.

GA-1 is thought to be rare in adults, but there are almost certainly unrecognised cases among populations of adults with stable neurological deficits acquired in childhood. This report illustrates the clinical and neuroradiological findings that may be seen in adults and extends the clinical range of GA-1.

M C PREVETT R S HOWARD Department of Neurology, St Thomas' Hospital, Guy's and St Thomas' NHS Trust, Lambeth Palace Road,

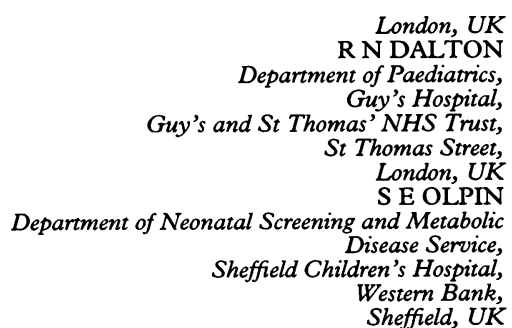

Correspondence to: Dr M Prevett, National Hospital for Neurology and Neurosurgery, Queen Square, London WCIN 3BG, UK

1 Goodman SI, Ferman FE. Organic acidemias due to defects in lysine oxidation: 2-ketoadipic acidemia and glutaric acidemia. In: Scriver CR, Beaudet AL, Sly WS, Valle D, eds. The metabolic basis of inherited disease. 6th ed. New York: McGraw-Hill, 1989:845-53.

2 Kyllerman M, Skjeldal OH, Lundberg M, et al. Dystonia and dyskinesia in glutaric aciduria type 1: clinical heterogeneity and therapeutic considerations. Mov Disord 1994;9:22-30.

3 Haworth JC, Booth FA, Chudley AE, et al. Phenotypic variability in glutaric aciduria Phenotypic variability in glutaric aciduria type 1: report of fourteen cases in five

4 Morton DH, Bennett MJ, Seargeant LE, Nichter CA, Kelley RI. Glutaric aciduria type 1: a common cause of episodic encephalopathy and spastic paralysis in the Amish of Lancaster County. Am $\mathcal{F}$ Med Genet 1991;41:89-95.

5 Lera G, Bhatia K, Marsden CD. Dystonia as the major manifestation of Leigh's syndrome. Mov Disord 1994;9:642-9.

The usefulness of functional imaging in movement disorders: an illustrative case

The clinical utility of functional imaging for movement disorders is limited but as pointed out in a review by Sawle ${ }^{1}$ it can be a valuable tool in the subset of patients where a diagnosis of dopa responsive dystonia versus young onset Parkinson's disease is suspected. These two conditions can be clearly differentiated by a $\left[{ }^{18} \mathrm{~F}\right]$-dopa scan..$^{2-4}$ The distinction between the two conditions is crucial with regard to therapy and prognosis. Because of the problems associated with levodopa therapy in young onset Parkinson's disease, ${ }^{5}$ an early diagnosis is helpful so that alternate treatment, such as the use of agonists, can be initiated keeping levodopa in reserve for as long as possible to delay the emergence of motor fluctuations and dyskinesiae. We report an illustrative case.

This 26 year old woman had normal birth and milestones. She first noticed problems at the age of 11 years when she began to walk on the outside of her feet with knock knees. At the age of 12 she developed a tremor of both arms and occasionally of her legs. As she was thought to have a spastic paraparesis, a myelogram was performed which was normal as was examination of the CSF. She had only a mild deterioration between the ages of 12 to 25 years, but then the tremor and her gait markedly worsened over a year. Her maternal grandfather and a maternal aunt were said to have had a similar tremor but further details were unknown. On examination she had parkinsonian features with an expressionless face, stooped posture, pronounced bradykinesia and rigidity. Her tendon reflexes were very brisk but the plantar responses were flexor. The rest of the neurological examination disclosed no other abnormality. Routine and special investigations including blood counts, serum biochemistry, and tests to exclude Wilson's disease were all normal. Head CT was normal. 